\title{
In-Situ White Beam Microdiffraction Study of the Deformation Behavior in Polycrystalline Magnesium Alloy During Uniaxial Loading
}

\author{
P. A. Lynch*, , A. W. Stevenson, D. Liang, D. Parry, S. Wilkins ${ }^{\dagger}$, I. C. Madsen*, C. \\ Bettles** and N. Tamura, G. Geandier $^{*}$ \\ *Commonwealth Scientific and Industrial Research Organisation (CSIRO), Minerals, Private Bag 33, Clayton \\ South MDC, 3169, Australia \\ ${ }^{\dagger}$ CSIRO, Manufacturing and Infrastructure Technology. \\ **ARC Centre of Excellence for Design in Light Metals, Department of Materials Engineering, Monash \\ University, Clayton, 3800, Victoria, Australia \\ ${ }^{\ddagger}$ Lawrence Berkeley National Laboratory, 1 Cyclotron Road, Berkeley, CA 94720, U.S.A.
}

\begin{abstract}
Scanning white beam X-ray microdiffraction has been used to study the heterogeneous grain deformation in a polycrystalline $\mathrm{Mg}$ alloy (MgAZ31). The high spatial resolution achieved on beamline 7.3.3 at the Advanced Light Source provides a unique method to measure the elastic strain and orientation of single grains as a function of applied load. To carry out in-situ measurements a light weight $(\sim 0.5 \mathrm{~kg})$ tensile stage, capable of providing uniaxial loads of up to $600 \mathrm{~kg}$, was designed to collect diffraction data on the loading and unloading cycle. In-situ observation of the deformation process provides insight about the crystallographic deformation mode via twinning and dislocation slip.
\end{abstract}

Keywords: white beam microdiffraction, plastic deformation, magnesium

PACS: PACS number required

\section{INTRODUCTION}

As a non-destructive test, $\mathrm{X}$-ray diffraction techniques are well suited to provide microstructural information during a dynamic process, as encountered in the standard engineering mechanical strength test. To gain a greater understanding of the bulk specimen microstructure, in-situ X-ray diffraction represents a routine method for measuring the residual stress and texture as a function of applied tensile or compressive load, see for example [1,2]. Most recently, high-spatial resolution microdiffraction synchrotron beamlines have been used to study the dynamic behavior of individual grains in a polycrystalline material under applied tensile load. Using a high energy focused monochromatic beam, Margulies et al.[3] have measured the lattice rotation of an individual grain under applied load. This technique has been further refined to study strain tensor development from individual [4] and multiple grains [5] in a polycrystalline sample under tensile load. In a different approach, using a highly focused synchrotron white beam, Joo et al. $[6,7]$ have used in-situ tensile measurements to study the deformation of individual copper grains. An underpinning requirement of these in-situ studies is the necessity to load the sample in incremental steps to enable data collection, either by specimen rotation or raster scanning the sample under the focussed incident beam. Both of these approaches provide a unique set of microstructural information from individual grains. Using focused synchrotron white or pink beam and appropriate sample positioning loading device, real time stress-strain microstructural information can be realised. The importance of this further development is that it will enable critical features of an engineering stress test, such as strain rate, to be measured on a grain and sub-grain level. Furthermore, the ability to monitor a region of interest during a loading or unloading cycle will provide a measurement scenario for pseudo-elastic deformation studies.

This article will outline the progress towards development of a real-time dynamical white beam microdiffraction experimental approach to measure the deformation of individual grains under load. The principle requirements of the deformation stage for performing high-spatial resolution microdiffraction experiments are discussed. This is followed by in-situ uniaxial tensile data collected for an $\mathrm{Mg}$ alloy sample at the microdiffraction beamline 7.3.3 at the Advanced Light Source. 


\section{EXPERIMENTAL}

\section{Deformation Stage Design}

To accommodate scanning microdiffraction requirements the deformation stage had to meet two key requirements. Firstly, due to weight constraints introduced by the high spatial resolution translation stages the total weight of the stage had to be restricted $(<0.5 \mathrm{~kg})$. Secondly, to ensure the incident beam is in close proximity to the sample, the physical size of the stage had to fit into a small volume of $\sim 20 \mathrm{~cm}$ length, $5 \mathrm{~cm}$ wide $\mathrm{x} 5 \mathrm{~cm}$ height. Consequently, standard commercially available tensile stages were inappropriate. In view of the experimental demands and sample requirements, a deformation stage capable of producing a tensile load of up to $6 \mathrm{kN}$ was designed and manufactured. The stage with $\mathrm{Mg}$ alloy 'dog bone' sample is shown in Figure 1.

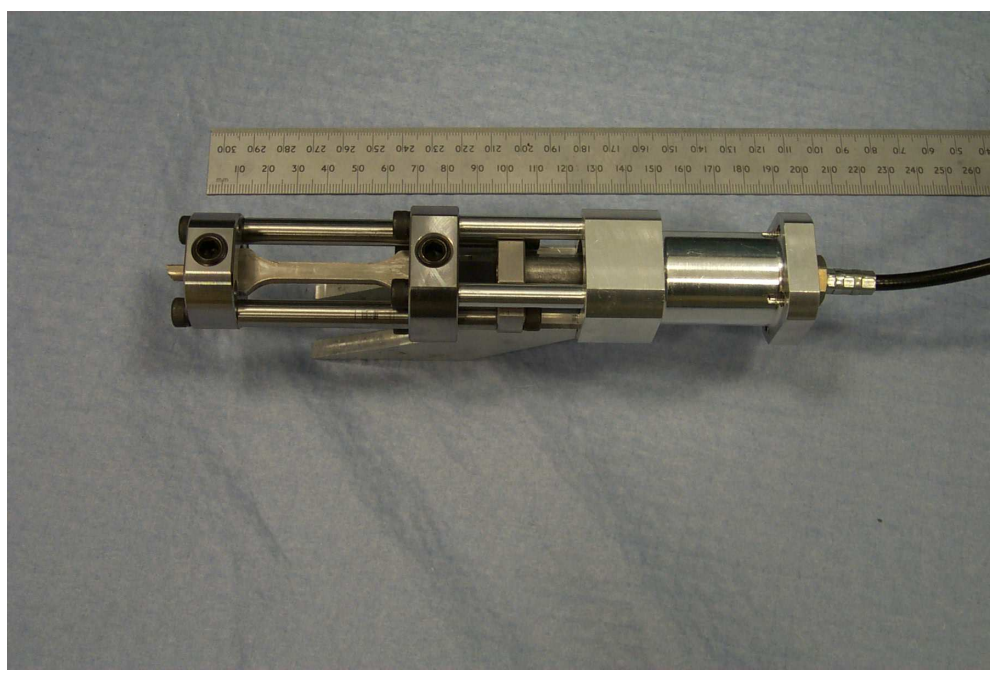

FIGURE 1. Hydraulic deformation stage configured for uniaxial tensile experiments.

The stage and hydraulic control system was designed as a fully portable device to allow for transfer to a synchrotron beamline end-station. For the current experimental requirements, the loading system configuration operates via a single-action hydraulic cylinder that provides a maximum tensile load of up to $6.0 \mathrm{kN}$. Sample loading and control is achieved by an input and output transducer connected to the hydraulic intensifier. The intensifier requires house or compressed air which is scaled according to the required sample load. All input and output transducer control is achieved with a portable analog pc card which uses a USB connection, enabling control with a laptop personal computer. Finally, loading cycles and feedback are achieved with a windows GUI interface.

\section{Microdiffraction Results and Analysis}

In-situ experiments were performed on the high-resolution synchrotron microdiffraction beamline (7.3.3) at the Advanced Light Source. For the present experiments the white beam from the bend magnet is focused using Kirkpatrick Baez mirrors to a spot size of $\sim 1 \mu \mathrm{m}^{2}$ onto the sample. For a detailed description of the beamline characteristics the reader is referred to MacDowell et al. [8]. Measurements were collected from a MgAZ31 (96\% Mg, 3\% Al, 1\% Zn) sample rolled to a thickness of $3.6 \mathrm{~mm}$. After a post-rolling anneal the sample was cut into the form of a regular 'dog bone' shape for tensile testing.

To conduct in-situ experiments the tensile stage was mounted on an XYZ translation stage, the sample was then positioned in a symmetric diffraction geometry with the incident beam angle $\omega=45^{\circ}$ and CCD detector set at $90^{\circ} 2 \theta$. To test the stability of the tensile stage, diffraction data was collected using a raster scan approach; the sample was translated across the beam in $8 \mu \mathrm{m}$ steps, generating an XY diffraction map from a specific region in the sample. A total of 7 diffraction maps were collected in load increments of $0.36 \mathrm{kN}$, ranging from 0 to $2.16 \mathrm{kN}$.

Indexation of the collected white beam patterns was achieved using the software package XMAS [9]. Figure 2 shows an example of the in-situ grain orientation maps collected for the MgAZ31 sample at load increments of $0.36 \mathrm{kN}$, 
$0.72 \mathrm{kN}$ and $1.8 \mathrm{kN}$. Figure $2 \mathrm{a}, \mathrm{c}$, e represents the out-of-plane orientation of [0002] relative to the surface normal and Fig. $2 b, d, f$ shows the in-plane orientation of [1010] relative to the scanning $X$ direction, where the $\mathrm{X}$ and $\mathrm{Y}$ directions are parallel and perpendicular to the tensile axis respectively. The dashed box in each figure highlights the same grain region for each data set. By tracking this region in each load increment the deformation of 5 grains could be recorded.

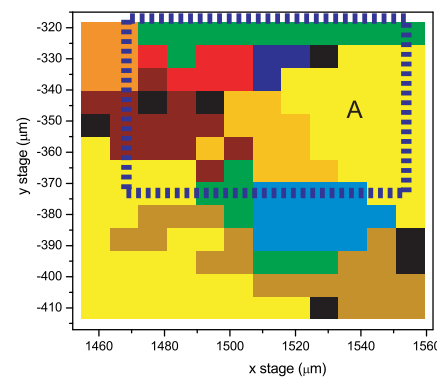

a).

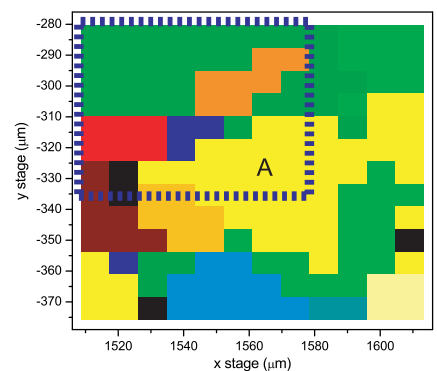

c).

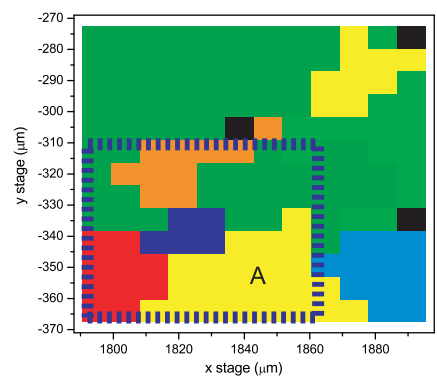

e).
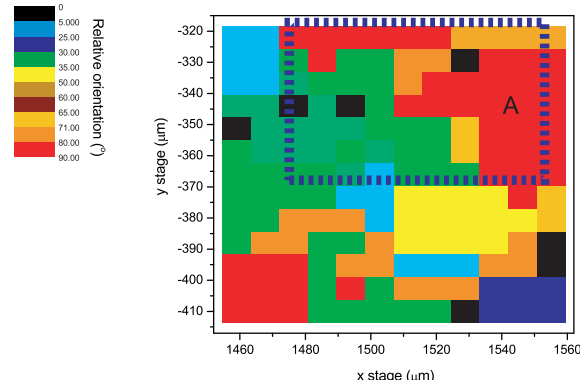

b).
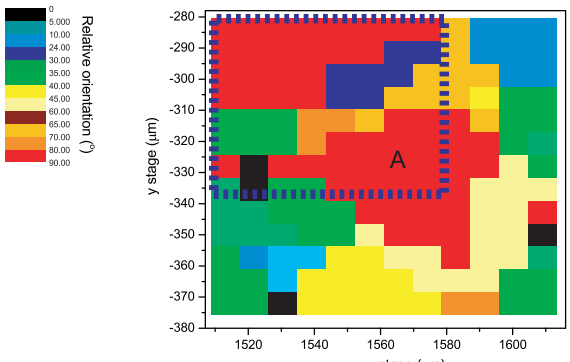

d).
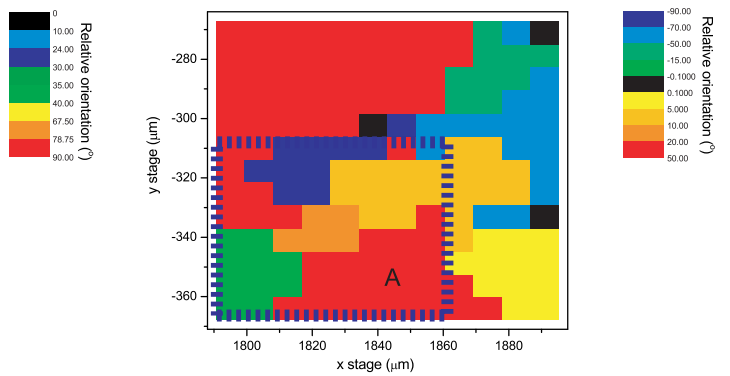

f)

FIGURE 2. Grain orientation maps of the MgAZ31 sample at load increments of $0.36 \mathrm{kN}(\mathrm{a}, \mathrm{b}), 0.72 \mathrm{kN}(\mathrm{c}, \mathrm{d})$, and $1.80 \mathrm{kN}(\mathrm{e}, \mathrm{f})$. The dashed box for each figure is used to highlight the same region on the sample at each load increment.

As an example, the deviatoric strain in the $\mathrm{X}$ and $\mathrm{Y}$ directions and the in-plane rotation angle have been plotted for Grain A in Figure 3. As illustrated in Figure 3a the applied load is observed as an increase in the tensile strain parallel to the load axis and compressive strain perpendicular to the load axis. For the in-plane rotation angle (Figure $3 b)$ only a relatively small shift between the [1010] and tensile axis was seen. In similar in-situ microdiffraction tensile experiments performed on FCC structures [6] an in-plane lattice rotation of up to $10^{\circ}$ was reported. Grain lattice rotation was attributed to the active slip system in the FCC structure. In this study it is proposed that the limited slip geometry in the hcp structure may constrain the in-plane lattice rotation and consequently induced lattice deformation is relieved via deformation twinning. To test this hypothesis an algorithm is presently under development which will provide information regarding the nature and content of deformation twinning in the sample. 


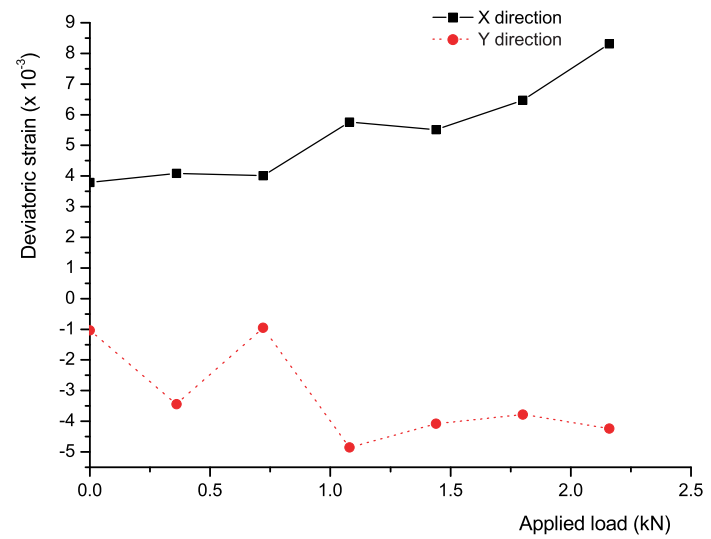

a).

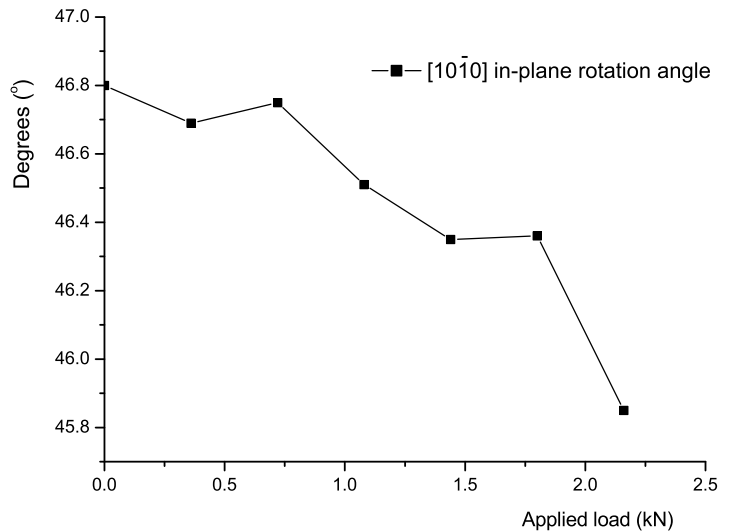

b).

FIGURE 3. The observed deformation of grain A as a function of applied load. a). Deviatoric strain parallel and perpendicular to the load axis. b). Change of the in-plane angle between the [1010] and load axis.

\section{CONCLUSION}

In this investigation a light weight strain stage has been successfully used to study the in-situ deformation of individual grains during uniaxial tensile loading. Using a raster scan approach it was possible to monitor the grain orientation and deviatoric strain as a function of applied load. The outcome of this preliminary work lends strong support for the future development of real time dynamic deformation studies based on white beam microdiffraction. This dynamic process can be realised with grain tracking strain stage development, currently in progress.

\section{ACKNOWLEDGMENTS}

We would like to thank K. Venkatesan and M. Gibson for scientific discussions and assistance with sample preparation. This work was supported by the Victorian Centre for Advanced Materials Manufacturing (VCAMM). The Advanced Light Source is supported by the Director, Office of Science, Office of Basic Energy Sciences, of the U. S. Department of Energy under Contract No. DE-AC02-05CH11231.

\section{REFERENCES}

1. S. R. Agnew, M. H. Yoo, and J. A. Horton, Magnesium Alloy pp. 119- 124 (2000).

2. D. W. Brown, S. R. Agnew, M. A. M. Bourke, T. M. Holden, S. C. Vogel, and C. N. Tome, Mat. Sci. Eng. A 399, 1-12 (2005).

3. L. Margulies, G. Winther, and H. F. Poulsen, Science 291, 2392-2394 (2001).

4. L. Margulies, T. Lorentzen, H. F. Poulsen, and T. Leffers, Acta Mat. 50, 1771-1779 (2002).

5. R. V. Martins, L. Margulies, S. Schmidt, H. F. Poulsen, and T. Leffers, Mat. Sci. Eng. A 387-389, 84-88 (2004).

6. H. D. Joo, J. S. Kim, K. H. Kim, N. Tamura, and Y. M. Koo, Scripta Mat. 51, 1183-1186 (2004).

7. H. D. Joo, J. S. Kim, C. W. Bark, J. Y. Kim, Y. M. Koo, and N. Tamura, Mat. Sci. For. 475-479, 4149-4152 (2004).

8. A. A. MacDowell, R. S. Celestre, N. Tamura, R. Spolenak, B. Valek, W. L. Brown, J. C. Bravman, H. A. Padmore, B. W. Batterman, and J. R. Patel, Nucl. Inst. Meth. Phys. Res. A 467-468, 936-943 (2001).

9. N. Tamura, A. A. MacDowell, R. Spolenak, B. C. Valek, J. C. Bravman, W. L. Brown, R. S. Celestre, H. A. Padmore, B. W. Batterman, and J. R. Patel, J. Syn. Rad. 10, 137-143 (2003). 